\title{
Changes in Microtubule Number and Length during Axon Differentiation
}

\author{
Wenqian Yu and Peter W. Baas \\ Department of Anatomy, The University of Wisconsin Medical School, Madison, Wisconsin 53706
}

\begin{abstract}
Hippocampal neurons in culture initially extend several minor processes that are $\approx 20 \mu \mathrm{m}$ in length. The first minor process to grow $\approx 10 \mu \mathrm{m}$ longer than the others will continue to grow rapidly and become the axon (Goslin and Banker, 1989). We sought to define changes in the microtubule (MT) array that occur during axon differentiation. In theory, axon differentiation could involve an increase in MT number, MT length, or some combination of both. To address this issue, we first serially reconstructed the entire $M T$ array of a minor process from a cell whose axon had not yet differentiated. This minor process contained $182 \mathrm{MTs}$ that ranged in length from 0.14 to $20.09 \mu \mathrm{m}$. The average MT length was $3.87 \pm 3.83 \mu \mathrm{m}$, and the total MiT length was $704 \mu \mathrm{m}$. We then reconstructed the MT arrays of a minor process and the $56 \mu \mathrm{m}$ axon from a cell that had undergone axon differentiation. The minor process contained 157 MTs that ranged in length from 0.24 to $17.95 \mu \mathrm{m}$. The average MT length was $3.91 \pm 4.84 \mu \mathrm{m}$, and the total MT length was $600 \mu \mathrm{m}$. The axon contained 1430 MTs that ranged in length from 0.05 to $40.14 \mu \mathrm{m}$. The average MT length was $4.02 \pm 5.28 \mu \mathrm{m}$, and the total MT length was $5750 \mu \mathrm{m}$. These data indicate that a shift occurs toward shorter as well as longer MTs, but that virtually no change in average MT length occurs during axon differentiation. Thus, elongation of existing MTs cannot account for the major expansion of the MT array that occurs as a minor process becomes an axon. In contrast, the number of MTs increases by $\approx \mathbf{1 0}$-fold as a minor process differentiates and grows into an axon of the length we analyzed. Based on these data, we conclude that the MT array of a minor process is substantially expanded as it differentiates into an axon, and that the principal mechanism by which this expansion occurs is the copious addition of new MTs.
\end{abstract}

IKey words: hippocampal neuron, axon, minor process, microtubule assembly, microtubule transport, neuronal development]

Neurons have complex morphologies that result from an intricate program of developmental events. Before the establishment of elaborate dendritic arbors, typical neurons extend a single axon that grows rapidly toward its target tissue. The differen-

\footnotetext{
Received Aug. 19, 1993; revised Oct. 14, 1993; accepted Oct. 26, 1993.

We thank F. J. Ahmad for assistance in the preparation of the figures. This work was funded by National Institutes of Health Grant NS 28785 and National Science Foundation Grant IBN-9209939 to P.W.B., who is also the recipient of a Research Career Development Award from the National Institutes of Health.

Correspondence should be addressed to Dr. Peter W. Baas, Department of Anatomy, The University of Wisconsin Medical School, 1300 University Avenue, Madison, WI 53706

Copyright (c) 1994 Society for Neuroscience $0270-6474 / 94 / 142818-12 \$ 05.00 / 0$
}

tiation of the axon represents the initial polarization of the neuron, and is therefore a milestone in the development of the neuron. For this reason, axon differentiation has been a matter of great interest. Understanding of this and other issues concerning neuronal development has been significantly enhanced in recent years through the use of cultures of embryonic rat hippocampal neurons (for reviews, see Banker and Waxman, 1988; Goslin and Banker, 1991). Upon plating, these neurons undergo a stereotyped sequence of developmental events that presumably recapitulates certain key features of neuronal development within the animal (Dotti et al., 1988). Initially, the neurons extend lamellipodia (stage 1), after which the lamellipodia coalesce into several essentially similar minor processes (stage 2). One of the minor processes then differentiates into the axon (stage 3), after which those remaining differentiate into dendrites (stage 4). The events underlying the differentiation of the axon during stage 3 are unknown, but it is clear that any of the minor processes has the potential to develop into the axon (Dotti and Banker, 1987). Additional work indicates that the first minor process to grow roughly $10 \mu \mathrm{m}$ longer than the others will continue to grow rapidly and become the axon (Goslin and Banker, 1989).

To better understand the cellular and molecular events underlying axon differentiation, we have focused in the present study on changes in the microtubule (MT) array that occur as a minor process differentiates into the axon. MTs are of particular interest in neuronal development and axon differentiation for two reasons. First, MTs are prominent architectural elements that provide structural support essential for axon initiation and growth. Second, MTs act as railways along which cytoplasmic organelles such as mitochondria and synaptic vesicle precursors are transported. Because different types of organelles tend to move along the MT specifically toward either its plus or minus end, the uniformly plus-end-distal orientation of MTs in the axon is a major determinant of its cytoplasmic composition (Baas et al., 1988; Black and Baas, 1989). Based on these observations, it seems reasonable that the MT array of one of the minor processes may undergo substantive alterations during stage 3 that are critical to its differentiation into the axon. Unfortunately, the nature of these alterations has not been forthcoming. For example, no change in MT polarity orientation occurs as a minor process develops into the axon (Baas et al., 1989), the position of the centrosome appears to be irrelevant to axon differentiation (Dotti and Banker, 1991), and no changes in tubulin modifications indicative of MT stability predict which minor process extended by a cultured hippocampal neuron will become the axon (Dotti and Banker, 1991).

Nevertheless, at least one major change in the MT array during axon differentiation appears to be certain; namely, that it 
expands profoundly. For example, as a minor process becomes the axon and lengthens by roughly triple, there is nearly a 10fold increase in MT mass within the process (present results). Given the key functions that MTs serve within the axon, it seems likely that this profound increase in MT mass is a defining event. Mechanistically, three possibilities seem worthy of consideration. First, the expansion in MT mass may result from the lengthening of existing MTs within the minor process. Alternatively, there may be an addition to the process of a great many new MTs such that the number of MTs increases dramatically. Finally, axon differentiation may result from some combination of increases in both MT number and length. To resolve this issue and better understand the mechanism by which the MT array of a minor process expands during axon differentiation, we have serially reconstructed the MT arrays of a minor process from a cultured hippocampal neuron in stage 2 , and a minor process and the axon from a neuron early in stage 3 . From these studies, we have obtained complete data on the number of MTs and length of each MT within these processes.

\section{Materials and Methods}

Cell culture. Cultures of embryonic rat hippocampal neurons were prepared as previously described (Goslin and Banker, 1991). Briefly, hippocampi were dissected from $18 \mathrm{~d}$ rat embryos, treated with trypsin for $15 \mathrm{~min}$, dissociated with trituration, and plated at a density of $\approx 1000$ cells $/ \mathrm{cm}^{2}$ in Minimum Essential Medium (GIBCO-Bethesda Research Labs, Grand Island, NY) containing 10\% horse serum. The cells were plated onto either glass coverslips or pieces of plastic broken from the bottoms of tissue culture dishes. In either case, the glass or plastic had been treated with polylysine prior to plating the cells. After $4 \mathrm{hr}$ of attachment, the neuron cultures were inverted into plastic tissue culture dishes containing a monolayer of astroglial cells. At this point, the medium consisted of Minimum Essential Medium, the N2 supplements described by Bottenstein (1985), $1 \mathrm{~mm}$ sodium pyruvate, and $0.1 \%$ ovalbumin. In cultures permitted to grow past the second day, cytosine arabinoside was added to the cultures on the third day to a final concentration of $5 \times 10^{-6} \mathrm{M}$ to inhibit the proliferation of non-neuronal cells.

Electron microscopy. In preparation for electron microscopy, the neuron cultures were fixed at $37^{\circ} \mathrm{C}$ in $0.1 \mathrm{~m}$ cacodylate containing $2 \%$ glutaraldehyde. Cultures from the same plating were fixed on different days so that we could examine neurons at different stages of development, and subsequent preparation for electron microscopy was similar to that described by Banker and Goslin (1991). Briefly, after 20 min of fixation, the cultures were rinsed twice for $5 \mathrm{~min}$ each in $0.1 \mathrm{M}$ cacodylate, postfixed for $5 \mathrm{~min}$ in $1 \% \mathrm{OsO}_{4}$, rinsed twice for $2 \mathrm{~min}$ in $\mathrm{NaCl}$, rinsed twice for $2 \mathrm{~min}$ in water, contrasted for $30 \mathrm{~min}$ in $5 \%$ aqueous uranyl acetate, dehydrated in ethanols, and embedded in LX-112 (Ladd, Burlington, VT). After curing overnight at $60^{\circ} \mathrm{C}$, the glass coverslip or tissueculture plastic on which the cclls had becn grown was removed from the resin by exposure to liquid nitrogen. For enhancement of their light microscopic appearance, embedded cultures were stained at $60^{\circ} \mathrm{C}$ for $30 \mathrm{~min}$ with $1 \%$ toluidine blue. Cells of interest were circled with a diamond marker objective and their images were recorded using a videoprinter (Sony Corporation, Japan). Thin sections with a uniform thickness of $100 \mathrm{~nm}$ were obtained with an Ultracut S Ultramicrotome (Reichert-Jung, Vienna, Austria), picked up on Formvar-coated slot grids, stained with uranyl acetate and lead citrate, and observed with a CX100 electron microscope (JEOL USA, Inc., Peabody, MA). Overlapping photographs at a magnification of $14,000 \times$ were obtained of each section through the entire minor process or axon of interest. In addition, photographs were taken of all sections through the centrosome.

Serial reconstruction and data analysis. Because the thin sectioning required for electron microscopy fragments MTs among many sections, it was necessary for determination of true MT lengths to reconstruct serially the entire MT array of processes from stage 2 and early stage 3 neurons. The strategy for accomplishing this was based on our previously described method (Baas and Heidemann, 1986; Joshi et al., 1986; Baas and Joshi, 1992). All MT profiles were traced from the electron micrographs together with membranous borders and some internal membranous elements onto transparent cellophane sheets. At this point, in our previous work, MT profiles were aligned with one another using the membranous borders and elements as registration markers, and the MTs were reconstructed and traced onto a composite drawing. However, because the MTs that normally occupy a three-dimensional neuronal process become superimposed upon one another in the preparation of a two-dimensional reconstruction, this part of the method was modified for better accuracy. The two ends of each MT profile in each section were assigned coordinates, based on their distance from a line through the hillock region drawn precisely where the process joins the cell body. These coordinates, together with the position of the MT profiles relative to the membranous borders of the process, were then used to trace individual MTs through progressive sections. MT profiles in two different sections were judged to be part of the same MT when the proximal end of one MT profile and the distal end of the other MT profile were spaced within two MT diameters $(\approx 0.05 \mu \mathrm{m})$ of one another with regard to all axes. In relatively rare $(<5 \%)$ cases of ambiguity regarding whether two MT profile were parts of the same or different MTs, the MT profiles were scored as part of the same MT. Data were tabulated in terms of the starting and stopping points for the entire lengths of individual MTs within the process. From these data, MT number, MT length ranges, average MT length, and total MT length were scored for the entire process and for different regions of the process. In the case of stage 4 axons, which are hundreds of micrometers long, it was impractical to undertake complete serial reconstruction. However, to obtain some sense of MT lengths in these axons, we used the abbreviated technique of Bray and Bunge (1981). For this, we reconstructed a $12 \mu \mathrm{m}$ region of axon that was hundreds of micrometers away from any cell body or discernable growth cone in the culture, and drew inferences on MT length based on the lengths of polymer exlending through this region.

\section{Results}

As noted in the introductory remarks, embryonic rat hippocampal neurons in culture undergo a stereotyped sequence of developmental events (Dotti et al., 1988). Initially the neurons extend lamellipodia (stage 1), which coalesce into minor processes that are $\approx 20 \mu \mathrm{m}$ in length (stage 2). One of the minor processes then starts growing more rapidly than the others and becomes the axon (stage 3 ). The transition between stages 1 and 2 generally occurs during the first day in culture, while the transition between stages 2 and 3 generally occurs during the second day in culture. For our purposes, we have defined "early stage 3 " as the period after axon differentiation but before the axon exceeds $100 \mu \mathrm{m}$ in length. Examples of neurons in stages 1 through early stage 3 are shown in Figure 1. The fact that there is some asynchrony among the cells in their rates of development is evidenced by Figure $1 c$, which shows one cell in stage 2 and another in early stage 3 . Because we were concerned in the present study with events underlying axon differentiation, we focused on cells in stage 2 and early stage 3 . Previous studies indicate that one of the minor processes becomes the definitive axon when its length exceeds $\approx 10 \mu \mathrm{m}$ that of the others (see Goslin and Banker, 1989). Nevertheless, we selected for our analyses axons that had become at least $40 \mu \mathrm{m}$ long to ensure that they had taken on clear axonal characteristics.

\section{Microtubule organization during axon differentiation}

In a first set of studies, we used standard electron microscopy to examine MT organization in stage 2 and early stage 3 neurons. Ten neurons at each stage were sectioned in their entirety so that we could analyze key regions such as the growth cones, shafts, and hillock regions of both minor processes and axons. We also examined MT organization within the cell body, focusing on the region around the centrosome and the regions continuous with the hillocks. No marked differences in MT organization among the different minor processes were observed, and this was true regardless of whether the minor process 

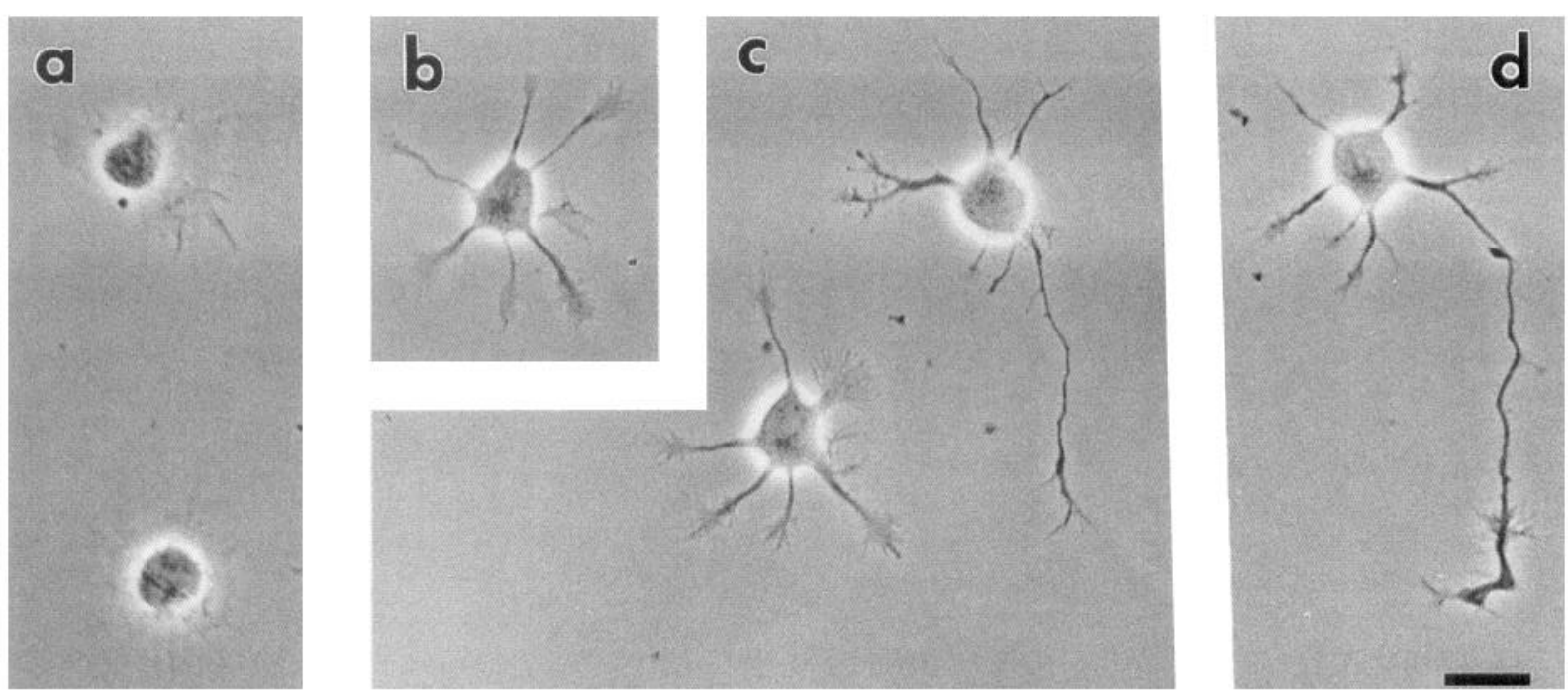

Figure 1. Phase-contrast micrographs of embryonic rat hippocampal neurons in culture at different stages of development. $a$ shows neurons in stage 1 , during which lamellipodia are extended. $b$ shows a neuron in stage 2 , during which several essentially similar minor processes are extended. $c$ shows another cell in stage 2 and a neighboring cell that has just entered stage 3 , during which one of the minor processes begins to elongate faster than the others and becomes the axon. Another cell in early stage 3 is shown in $d$. Scale bar, $15 \mu \mathrm{m}$.

was from a neuron in stage 2 or early stage 3 . By comparison, however, the axon was notably different from the minor processes with regard to MT organization. Specifically, the MTs in the minor process were spaced farther apart than those in the axon, and the spacing at different points along two adjacent
MTs varied more in the minor process compared to the axon. Figure $2 a$ shows a portion of the cell body continuous with the hillock and minor process from a stage 2 neuron, while Figure $2 c$ shows comparable regions of the cell body and axon from an early stage 3 neuron. The average spacing between MTs
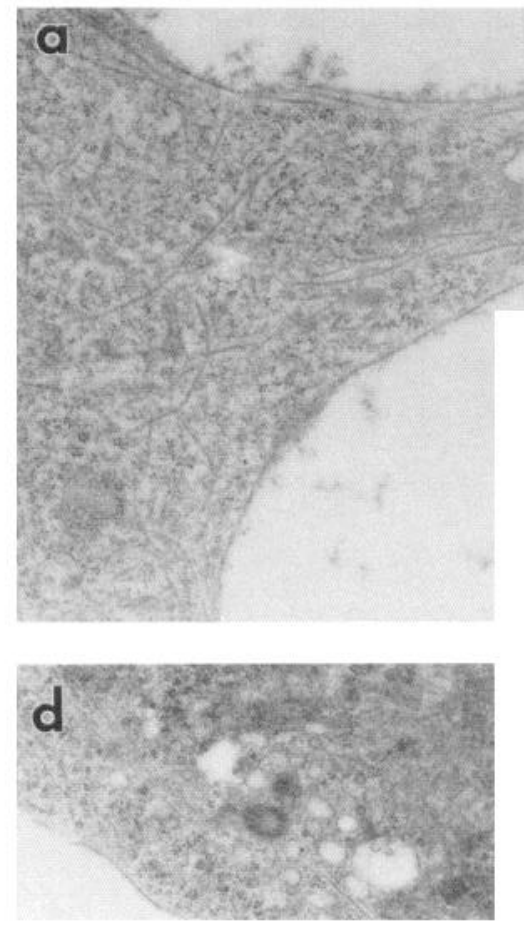
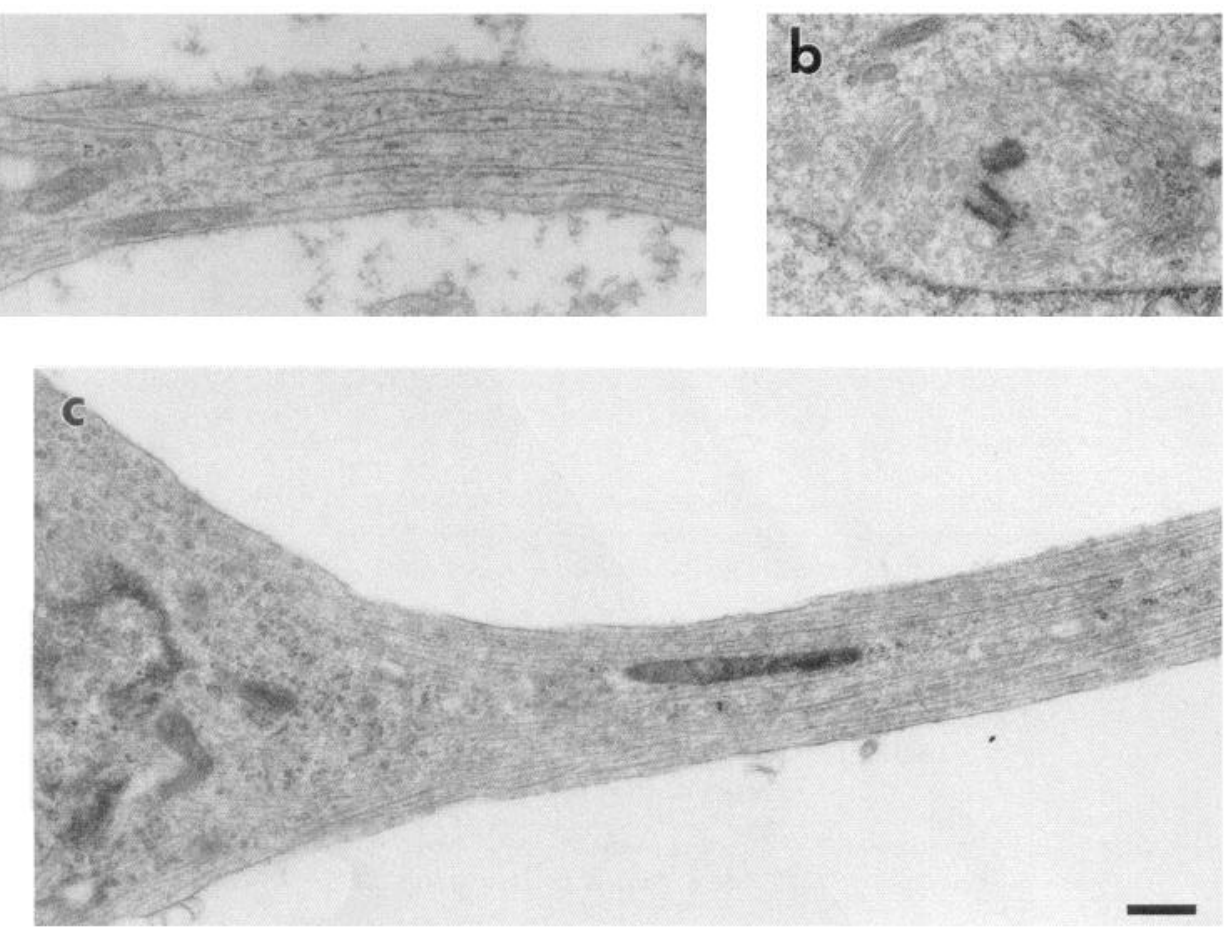

Figure 2. Electron micrographs of regions of a cultured hippocampal neuron in stage $2(a$ and $b)$, and early stage $3(c$ and $d)$. $a$ shows a portion of the cell body, a minor process, and the hillock region joining them. $c$ shows comparable regions of the cell body, hillock, and axon. MTs in the axon are spaced about twice as closely as those in the minor process, and are far more parallel to one another and consistent in their spacing. By comparison, neighboring MTs in the minor process vary in distance along their lengths, sometimes running at sharp angles to one another. This difference in organization is particularly accentuated in the hillock region. $b$ and $d$ show the centrosomal regions from these stage 2 and 3 neurons, respectively. Similar to our previous reports on the centrosomes of cultured sympathetic neurons (Baas and Joshi, 1992; Yu et al., 1993), few or no MTs are directly attached to these centrosomes. Scale bar, $0.5 \mu \mathrm{m}$. 

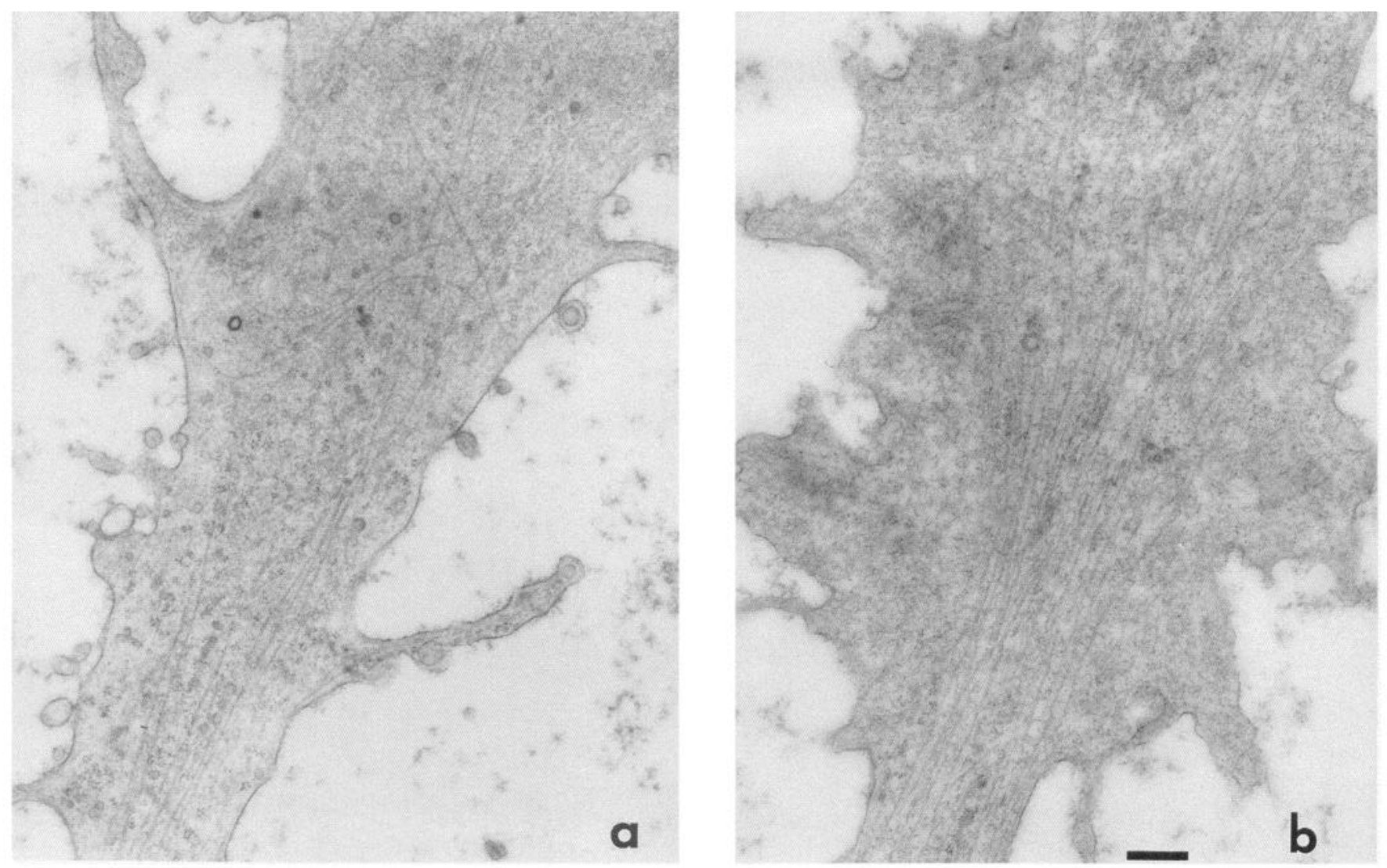

Figure 3. Electron micrographs of the growth cone region of a minor process from a stage 2 neuron $(a)$, and the axon $(b)$ from a stage 3 neuron. Although the cones are of generally similar morphology, the MT arrays within them differ markedly. Whereas the MTs in the axonal growth cone are relatively parallel to one another, the MT array of the minor process growth cone is less orderly, with some MTs running near perpendicular to the long axis of the process. Scale bar, $0.5 \mu \mathrm{m}$.

(nearest neighbor edge to edge) was $\approx 50 \mathrm{~nm}$ for the minor process, and $\approx 15 \mathrm{~nm}$ for the axon. With regard to the cell bodies of stage 2 and 3 neurons, the centrosomal regions (Fig. 2b,d) were generally similar to one another, with few $(<10$ among all of the sections) or no MTs directly attached to the centrosome. The hillock regions of the minor processes were also similar to one another in stage 2 and 3 neurons, but the axon hillock was markedly different. The MTs in the axon hillock were generally aligned side by side and more or less parallel to one other as they extended from the cell body into the axon, while the MTs in the hillock of the minor processes were not aligned but rather crossed one another as well as the long axis of the minor process at various and often obtuse angles.

The differences in MT organization between minor processes and the axon were also reflected in their most distal regions contiguous with their growth cones. The morphology of the growth cone varied from process to process, with some processes showing elaborate flattened cones with many filopodia and other processes showing rounded more modest cones with few or no filopodia. Both morphologies were noted for both minor processes and axons, presumably reflecting the behavior of the growth cone at the point of fixation. To compare MT organization between the growth cones of axons and minor processes, it was necessary to compare growth cones of similar morphology. Figure 3 shows two growth cones with the more flattened morphology, one from a minor process of a stage 2 neuron and the other from the axon of a Stage 3 neuron. In the latter, MTs splayed apart as they entered the cone, but were generally aligned more or less parallel to one another and to the long axis of the axon. The MTs in the minor process also splayed apart as they entered the cone. However, reminiscent of the hillock region of a minor process, MTs within the growth cone of a minor process were situated at various and often obtuse angles to one another and to the long axis of the process.

\section{Microtubule number and length in minor processes and early axons}

The principal goal of the present study was to determine the number of MTs and the length of every MT within a minor process from a stage 2 neuron, and a minor process and the axon from an early stage 3 neuron. To accomplish this, we undertook serial reconstruction of the entire MT arrays of these processes. The shaft regions of the minor processes and axon comprised 6 and 10 thin sections, respectively, while the somewhat thicker hillock regions comprised 10 and 15 thin sections, respectively. After tracing the MT profiles of each section onto cellophane sheets, it became apparent that attempts to incorporate all of the tracings together into a composite drawing would mask in a blur of ink the ends of MTs required to determine their individual lengths. Instead, therefore, the starting and stopping points of each MT profile on each section were assigned coordinates based on their distance from the hillock, and MT profiles from different sections were determined to be parts of the same or different MTs based on these coordinates 


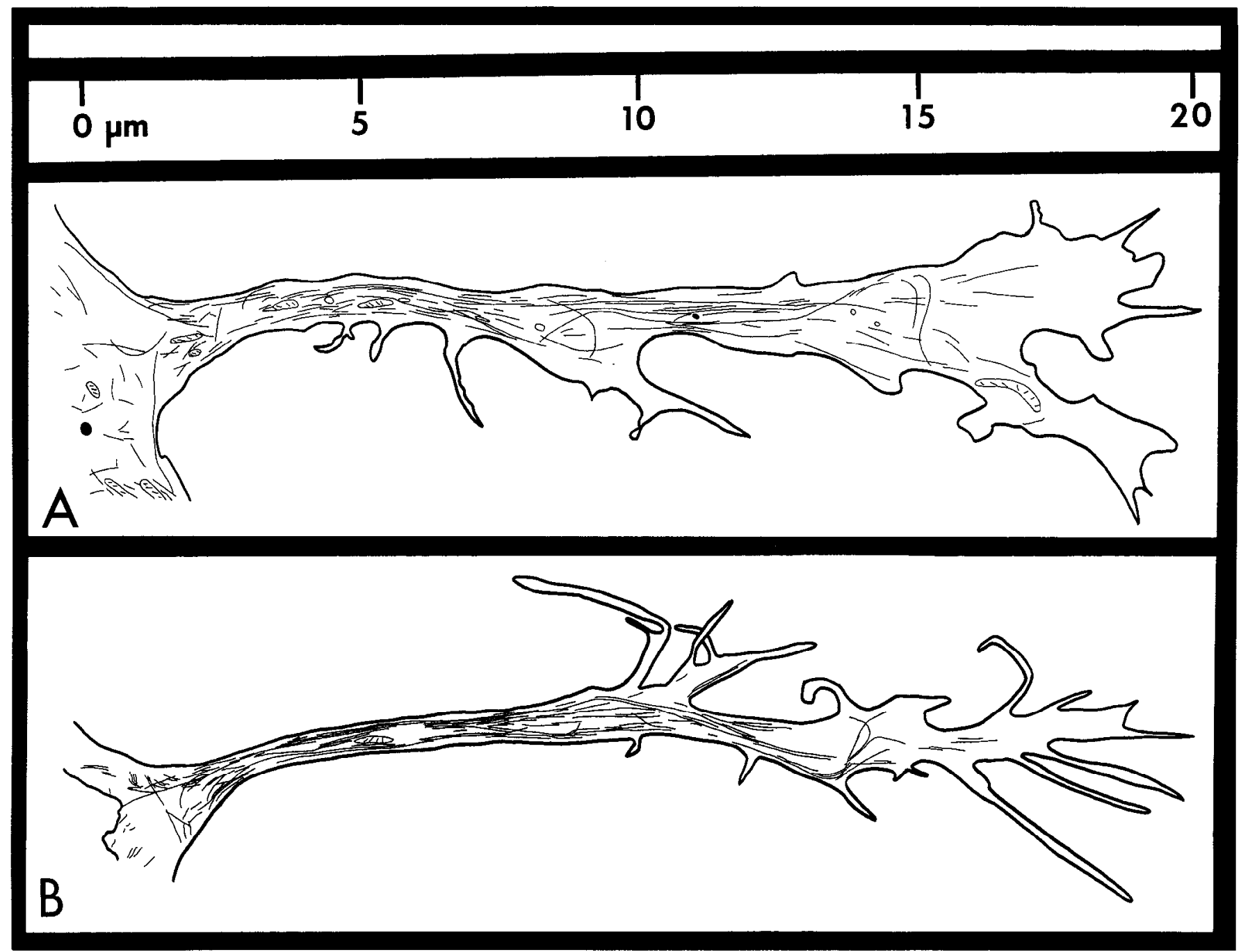

Figure 4. Tracings prepared from single sections through two different minor processes. The process shown in $A$ is from a stage 2 neuron, while the process shown in $B$ is from a stage 3 neuron. Because the MT array of the minor processes is fragmented during the thin sectioning required for electron microscopy, examination of a single section does not reveal true MT length or number. For serial reconstruction, we chose not to prepare composite figures, however, because the ends of MTs are generally lost in the complexity of such a figure. Instead, the ends of each MT profile in each section were assigned coordinates and these coordinates were used to calculate the length and position of each MT within the array. The tracing of a single section provides only a sense of our methodology, and the general organization of the MT array from the cell body to the growth cone. More details are provided in the Materials and Methods and Results. Data on MT length and number from these processes are provided in Table 1 . The scale bar at the top indicates length in micrometers.

(see Materials and Methods for more details). Figure 4, $A$ and $B$, shows tracings from single sections through the stage 2 and 3 minor processes, respectively. The former was $20.5 \mu \mathrm{m}$ and the latter was $19 \mu \mathrm{m}$ in length. Examination of single sections provides only an overview of the general organization of MTs from the cell body into the growth cone. Reconstruction of the complete MTs arrays of these processes revealed the following data, summarized in Table 1 . The stage 2 minor process contained 182 MTs that ranged in length from 0.14 to $20.09 \mu \mathrm{m}$. The average MT length was $3.87 \pm 3.83 \mu \mathrm{m}$, and the total MT length was $704 \mu \mathrm{m}$. The stage 3 minor process contained 157 MTs that ranged in length from 0.24 to $17.95 \mu \mathrm{m}$. The average MT length was $3.91 \pm 4.84 \mu \mathrm{m}$, and the total MT length was $600 \mu \mathrm{m}$. Histograms of the length distributions within the two minor processes reveal that $\approx 70 \%$ of the MTs were $4 \mu \mathrm{m}$ or less in length, and the numbers of longer MTs decrease progressively with greater length (see Fig. 5). These data indicate that stage 2 minor processes and stage 3 minor processes are generally similar to one another with regard to MT mass, length, and number.

Figure 6 shows a tracing from a single section through the axon from the same neuron, one of whose minor processes is shown in Figure $4 B$. The axon was $56 \mu \mathrm{m}$ long. Figure $6 A$ shows the axon at a magnification sufficiently low that its entire length can fit on the page, while Figure $6 B-D$ shows the axon divided into three parts shown at the same magnification as used for the minor processes in Figure 4. Again, only a general sense of MT organization can be gleaned from observation of a single section. Reconstruction of the complete MT array revealed that the axon contained 1430 MTs that ranged in length from 0.05 to $40.14 \mu \mathrm{m}$. The average MT length was $4.02 \pm 5.28 \mu \mathrm{m}$, and the total MT length was $5750 \mu \mathrm{m}$. These data, summarized in Table 1 , indicate that this $56 \mu \mathrm{m}$ axon contained nearly 10 times as much polymer as a minor process, and nearly 10 times as 

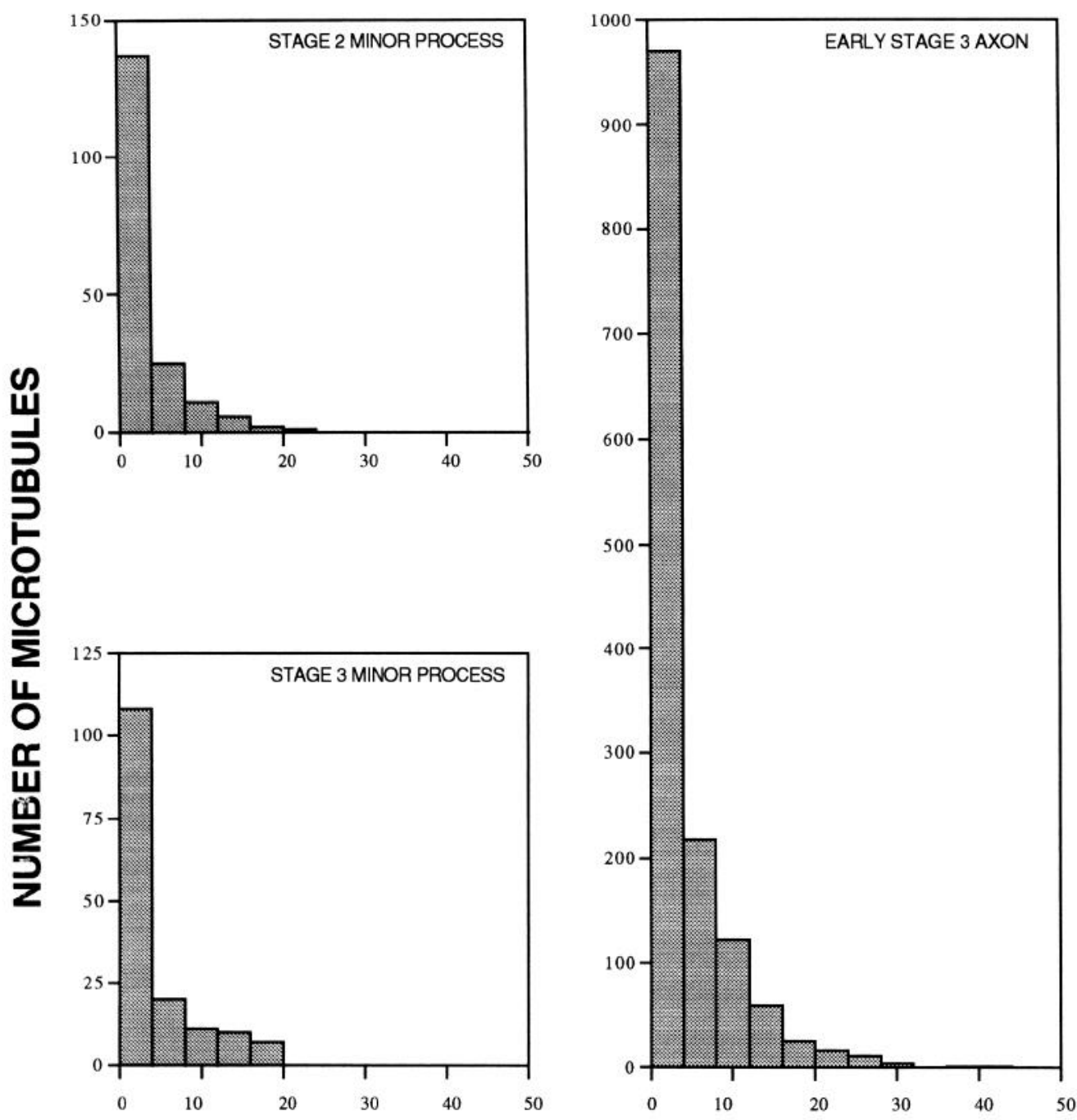

Figure 5. Histograms of the length distributions of MTs derived from serial reconstructions of the stage 2 minor process, the early stage 3 minor process, and the early stage 3 axon shown in MICROTUBULE LENGTH $(\mu \mathrm{m})$ Figures 4 and 6 . See Results for details.

many MTs. The histogram of the data is generally similar in appearance to that of the minor processes, with $\approx 70 \%$ of the MTs at $4 \mu \mathrm{m}$ or less and the remaining MTs decreasing in number at increasing lengths (Fig. 5). In addition though, the MT length range was greater in the axon, with longer MTs than found in the minor processes, and also shorter MTs (there were $122 \mathrm{MTs}$ in the axon equal to or shorter than $0.14 \mu \mathrm{m}$, and 213 MTs equal to or shorter than $0.24 \mu \mathrm{m}$, the shortest lengths found in the stage 2 and early stage 3 minor processes, respectively). Notably, however, the average MT length is virtually unchanged in the axon compared to the minor processes.

In terms of distribution along the process, virtually the entire length range of MTs was represented in all regions of the axon and the minor processes. The longest MTs in each process spanned from or close to the cell body into or close to the growth cone, and numerous other long MTs coursed from the proximal region deep into the shaft, or from deep within the shaft into the distal region near the growth cone. By contrast, the distribution of the shortest MTs was less even. In the stage 2 minor process, the early stage 3 minor process, and the early stage 3 axon, there were 79,56 , and $528 \mathrm{MTs}$, respectively, that were under $1 \mu \mathrm{m}$ in length, indicating fractions of the total number of MTs in each process of $43 \%, 36 \%$, and $37 \%$, respectively. In the stage 2 minor process, 57 of these short MTs were present in the most proximal $3 \mu \mathrm{m}, 8$ were present in the most distal 3 $\mu \mathrm{m}$, and 14 were present in the intervening $14.5 \mu \mathrm{m}$. In the

\begin{tabular}{|c|c|c|c|c|c|}
\hline & $\begin{array}{l}\text { Length } \\
\text { of } \\
\text { pro- } \\
\text { cess } \\
(\mu \mathrm{m})\end{array}$ & $\begin{array}{l}\text { Total } \\
\text { MT } \\
\text { length } \\
(\mu \mathrm{m})\end{array}$ & $\begin{array}{l}\text { Num- } \\
\text { ber } \\
\text { of } \\
\text { MTs }\end{array}$ & $\begin{array}{l}\text { MT length } \\
\text { range }(\mu \mathrm{m})\end{array}$ & $\begin{array}{l}\text { Average MT } \\
\text { length }(\mu \mathrm{m}, \\
\text { mean } \pm \mathrm{SD})\end{array}$ \\
\hline $\begin{array}{l}\text { Minor process } \\
\text { (stage 2) }\end{array}$ & 20.5 & 704 & 182 & $0.14-20.09$ & $3.87 \pm 3.83$ \\
\hline $\begin{array}{l}\text { Minor process } \\
\text { (stage } 3 \text { ) }\end{array}$ & 19 & 600 & 157 & $0.24-17.95$ & $3.91 \pm 4.84$ \\
\hline $\begin{array}{l}\text { Axon } \\
\text { (stage 3) }\end{array}$ & 56 & 5750 & 1430 & $0.05-40.14$ & $4.02 \pm 5.28$ \\
\hline
\end{tabular}




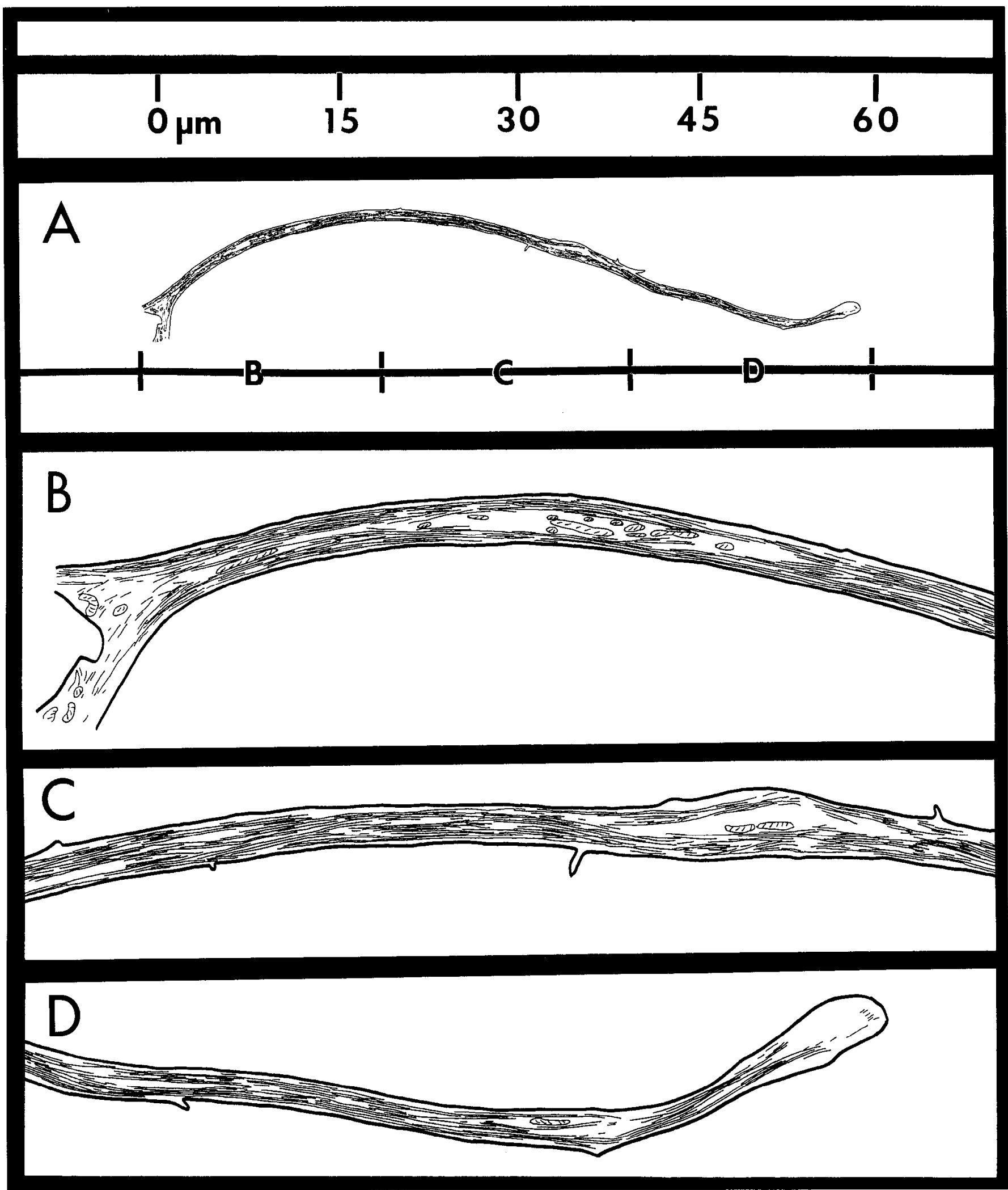

Figure 6. Tracing prepared from a single section through the axon from the same stage 3 neuron one of whose minor processes is shown in Figure $4 B$. As noted in the legend to Figure 4, we chose not to prepare composite serial reconstructions because most of the information on MT length and number was lost in the complexity of such a figure. Instead, the ends of each MT profile in each section were assigned coordinates and these coordinates were used to calculate the length and position of each MT within the array. More details are provided in the legend to Figure 4, and in the Materials and Methods and Results. Data on MT length and number from these processes are provided in Table $1 . A$ shows the tracing at a rather low magnification so that the entire length of the axon can fit on the page. The scale bar at the top indicates length in micrometers for $A$. Regions of the axon indicated by the letters $B-D$ are shown at higher magnification in the respective panels. 

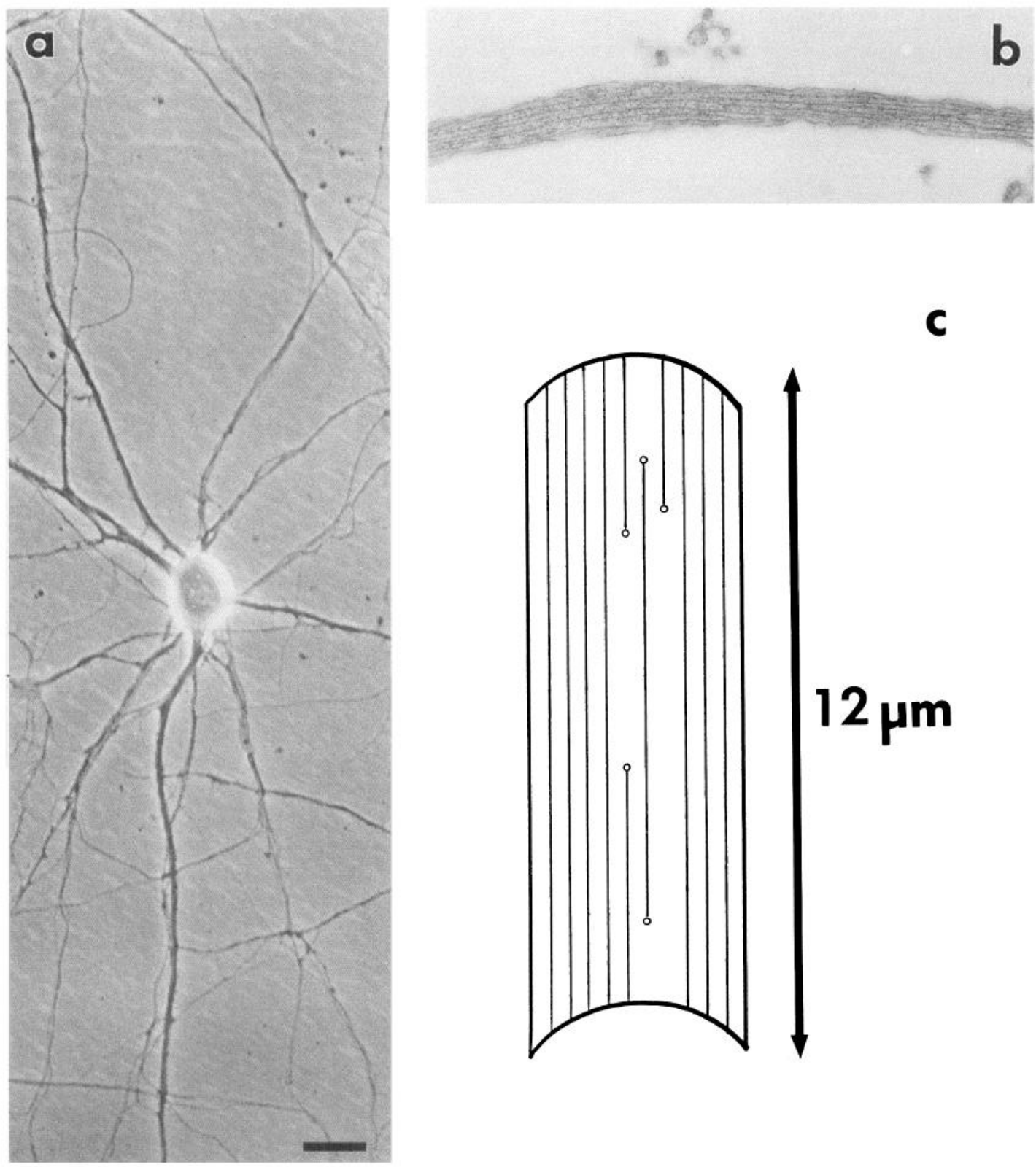

Figure 7. Summary of observations on stage 4 axons. $a$ is a phase-contrast image of a portion of a culture grown for $7 \mathrm{~d}$, in which the neurons have entered stage 4 . Extending from the cell body are thickened dendrites, which have lost their tapered appearance because they are fasciculated with portions of the dense axonal mat covering much of the culture dish. $b$ is an electron micrograph of a region of an axon taken from a portion of the dish hundreds of micrometers from any cell body or discernable growth cone. Compared to stage 3 axons, the stage 4 axons have generally become thinner and the number of MTs appearing in cross section is lower. $c$ shows a schematic summary of a serial reconstruction of the MT array within a $12 \mu \mathrm{m}$ region of this axon. The majority of MTs extend all of the way through the $12 \mu \mathrm{m}$ region, although five free MT ends were noted (open circles). See Results for more details. Scale bar: $15 \mu \mathrm{m}$ for $a, 0.6 \mu \mathrm{m}$ for $b$.

stage 3 minor process, 24 were present in the most proximal 3 $\mu \mathrm{m}, 4$ were present in the most distal $3 \mu \mathrm{m}$, and 28 were present in the intervening $13 \mu \mathrm{m}$. In the axon, 180 were present in the most proximal $3 \mu \mathrm{m}, 79$ were present in the most distal $3 \mu \mathrm{m}$, and 269 were present in the intervening $50 \mu \mathrm{m}$. If the short MTs were distributed evenly along the lengths of the processes, we would expect to find roughly 11,8 , and 28 MTs in $3 \mu \mathrm{m}$ intervals of the stage 2 minor process, early stage 3 minor process, and early stage 3 axon, respectively. These results indicate that there is some enrichment of the shortest MTs within the distal region of the stage 2 minor process, somewhat higher enrichment within the distal region of the early stage 3 axon, and markedly higher enrichment yet in the proximal regions of all three processes examined.

\section{Analyses on the microtubule array of stage 4 axons}

By stage 4 of development ( $7 \mathrm{~d}$ in culture), dendrites have developed and the axon has grown many times longer, weaving a complex path through the culture dish (Fig. 7a). In addition, the diameter of the axon and the number of MTs appearing in cross section have been reduced by about half relative to early stage 3 axons (Fig. $7 b$ ). It was impossible to follow an individual stage 4 axon from its cell body to its growth cone because of the complexity of the culture. However, based on the volume 
of the axonal mat, it is likely that typical stage 4 axons exceed $1000 \mu \mathrm{m}$ in length. Previous studies on cultured sensory neurons suggest that when their axons become this long, the average MT length probably exceeds $100 \mu \mathrm{m}$ (Bray and Bunge, 1981). To determine whether the MTs in the axons of cultured hippocampal neurons also continue to elongate as the axon grows, we used the same abbreviated method that Bray and Bunge used on the sensory axons (see Materials and Methods). Figure $7 c$ shows a schematic representation of a reconstruction of the MTs appearing in a $12 \mu \mathrm{m}$ stretch of the axon, taken from a region of the culture hundreds of micrometers from any cell body or discernable growth cone. The majority of the MTs (7 of 11) extended all of the way through the $12 \mu \mathrm{m}$ stretch, indicating that these MTs were at least $12 \mu \mathrm{m}$ long and potentially far longer. Three other MTs had one free end within the $12 \mu \mathrm{m}$ stretch. One MT had both ends within the $12 \mu \mathrm{m}$, and the true length of this MT was $8.5 \mu \mathrm{m}$. In general, however, this approach does not resolve the actual lengths of MTs within the axon. Nevertheless, the fact that most MTs extended through the 12 $\mu \mathrm{m}$, together with our lack of detection of "short" MTs (those under $1 \mu \mathrm{m}$ ), is consistent with the idea that many of the MTs within stage 4 axons take on lengths far greater than the lengths of the MTs within early stage 3 axons.

\section{Analyses on microtubule spacing in minor processes and axons}

In a final set of studies, we analyzed more carefully the spacing betwecn MTs in the same processes used for our scrial reconstruction work. For this, we measured the spacing (nearest neighbor edge to edge) at 500 randomly selected points between MTs within each of the arrays. The mean \pm SD for the stage 2 minor process, early stage 3 minor process, early stage 3 axon, and stage 4 axon was $54 \pm 38 \mathrm{~nm}, 48 \pm 39 \mathrm{~nm}, 15 \pm 14 \mathrm{~nm}$, and $43 \pm 8 \mathrm{~nm}$, respectively. Thus, the average spacing was similar in both minor processes and the stage 4 axon, but was significantly closer in the early stage 3 axon, presumably relating to the rapid increase in polymer levels accompanying axon differentiation. Also of note and consistent with our qualitative observations, in both the early stage 3 and stage 4 axons, the variability in spacing was far lower than that in the two minor processes, as indicated by histograms of the distribution of spacing distances between MTs (Fig. 8).

\section{Discussion}

In the present study, we sought to define changes in the MT array that occur as a minor process differentiates into the axon. Our data reveal that a typical minor process, $\approx 20 \mu \mathrm{m}$ in length, contains about 600-700 total $\mu \mathrm{m}$ of MT polymer, while an axon roughly three times as long contains almost 10 times as much polymer. Given the structural and functional importance of MTs, it seems likely that this increase in MT mass is a defining event in the differentiation of the axon. For this reason, we endeavored to understand the mechanism by which it occurs. In theory, axon differentiation could involve an increase in the length of the MTs within the minor process, an increase in the number of MTs, or some combination of both. To address this issue, we serially reconstructed the MT arrays within a minor process from a stage 2 neuron, and a minor process and the axon from an early stage 3 neuron. The strength of this approach is that it provides direct and complete information on the length and position of every MT within these processes, and does not rely on the kinds of inferences that would be inherent in less direct approaches. The weakness of serial reconstruction work on this level is that the sample number is necessarily limited by practicality. We are encouraged that our results are meaningful, however, because the MT arrays of the minor processes and the axon are so very different in terms of organization and mass. In addition, qualitative examination of serial sections through minor processes and axons from several additional neurons suggests that the three processes we reconstructed are generally representative of the processes extended by stage 2 and early stage 3 neurons.

Four broad conclusions can be drawn from our results. First, there are no notable differences between stage 2 and stage 3 minor processes with regard to any of the issues we studied. Second, the range of MT lengths increases during axon differentiation, with the appearance of many MTs that are longer than those in the minor processes as well as many MTs that are shorter. Third, despite these length changes, the average MT length within the axon is $\approx 4 \mu \mathrm{m}$, virtually unchanged from the average MT length within the minor processes. Finally, the most dramatic change in the MT array, and that which accounts for the nearly 10 -fold increase in MT mass, is a nearly 10 -fold increase in the total number of MTs as the process roughly triples in length. These results indicate that the major expansion of the MT array that occurs during axon differentiation is not the result of existing MTs simply getting longer. Rather, it is the copious addition of new MTs that accounts for this expansion.

\section{Mechanisms for elaborating the axonal microtubule array}

The present study represents part of our continuing efforts to understand the mechanisms by which the axonal MT array is elaborated. Although there is disagreement as to whether axon growth involves the transport of preassembled MTs from the cell body or local MT assembly within the axon, we have argued that axon growth is ultimately dependent upon both. The reasons for this view stem principally from consideration of the challenges that must be met by longer axons. Tubulin manufactured within the cell body must be translocated into the axon to support the expansion of its MT array. Diffusion in the subunit form could only supply sufficient levels of tubulin to support axon growth over the first few hundred micrometers, beyond which an active transport mechanism is required (Lasek, 1988). An abundance of kinetic data support the idea that tubulin is transported in the form of assembled MTs (for review, see Lasek, 1988), but for rcasons that arc unclear, efforts to observe MT movement in the axon with modern fluorescence techniques have not provided consistent results (Keith, 1987; Lim et al., 1989, 1990; Okabe and Hirokawa, 1989, 1992, 1993; Reinsch et al., 1991; Sabry et al., 1991; Tanaka and Kirschner, 1991; Keith and Farmer, 1993). However, several other lines of evidence support the view that MTs destined for the axon originate within the cell body, and hence by inference translucate from one compartment into the other. For example, when MT assembly in the neuron is pharmacologically inhibited, there is a gradual increase in MT polymer in the growing axon and a concomitant decrease in polymer from the cell body (Baas and Ahmad, 1993). In addition, other evidence from our laboratory suggests that no entirely new MTs are nucleated within the axon itself (Baas and Ahmad, 1992), and that MTs destined for the axon are probably nucleated and released from the centrosome within the cell body (Baas and Joshi, 1992; Yu et al., 1993). The need for local MT assembly within the axon is also clear, given the fact that many MTs in longer axons, such as the stage 4 axons we studied, attain lengths far greater than the diameter 

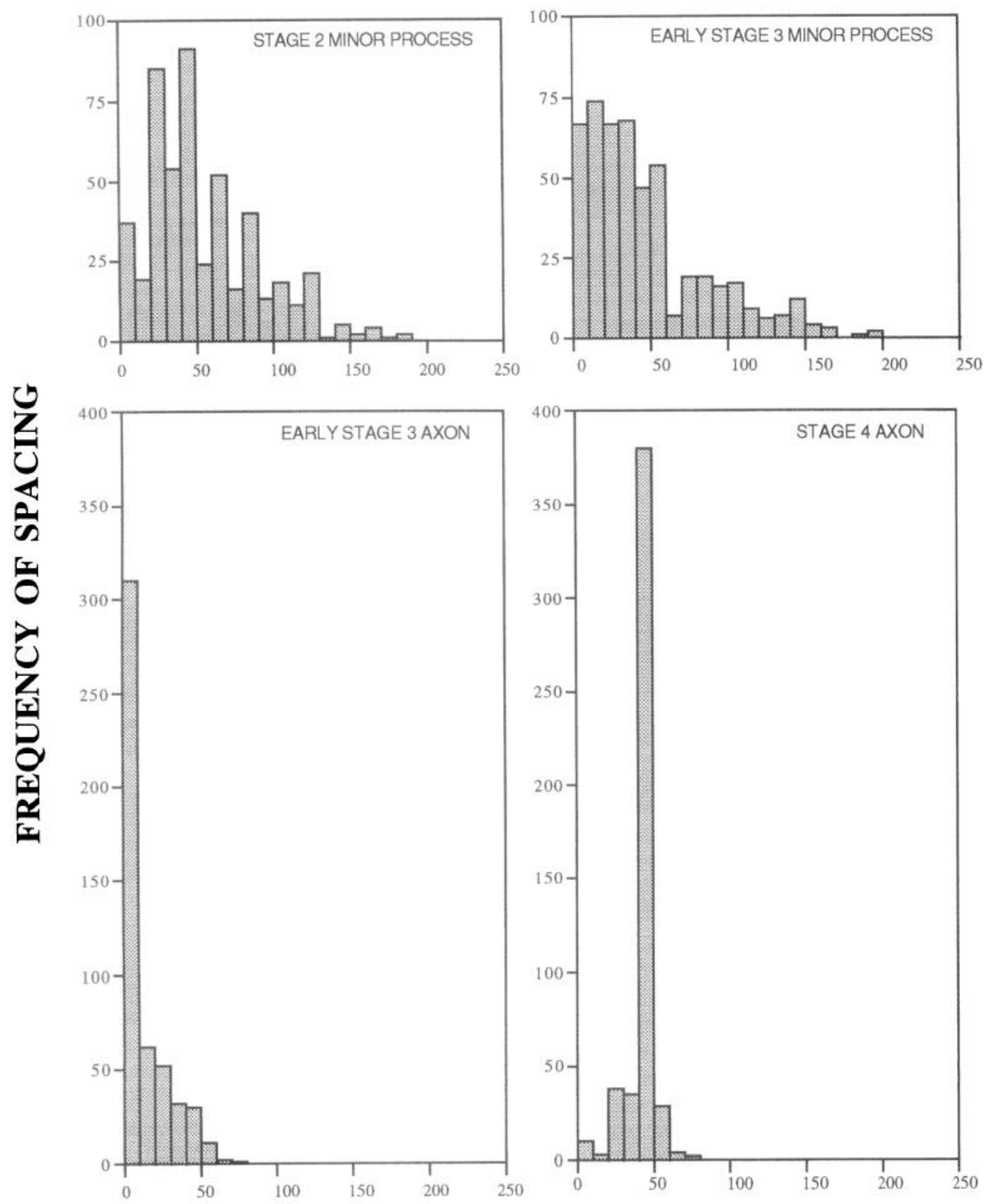

\section{SPACING BETWEEN MICROTUBULES (nm)}

Figure 8. Histograms of the spacing between MTs of the same stage 2 minor process, early stage 3 minor process, early stage 3 axon, and stage 4 axon used for the reconstruction analyses. See Results for details.

of the cell body (see also Bray and Bunge, 1981; Tsukita and Ishikawa, 1981; Burton, 1987).

Taking all of this into consideration, we have proposed a composite model that incorporates as key principals both MT transport and local assembly (Baas and Ahmad, 1993; Joshi and Baas, 1993). In this model, MTs are nucleated at the centrosome within the cell body, and then released. These MTs are short, a fraction of the diameter of the cell body, and many of them are translocated into the axon. The molecular machinery that transports the MTs has stereospecificity with regard to the polarity of the MT and thus sets up the uniformly plus-end-distal polarity orientation of axonal MTs (see Baas and Ahmad, 1993). During transit, many of the MTs shorten to provide subunits for the elongation of others, resulting in a shift toward longer and shorter MTs (as originally proposed by Lasek, 1988). Such a redistribution of MT lengths within a population is a key prediction of the dynamic instability model for MT behavior in living cells (for review, see Kirschner and Mitchison, 1986). Thus, our composite model for the elaboration of the axonal MT array is attractive in that it explains data previously used to support other models, incorporates principals of dynamic instability, and also satisfies the unique challenges of longer axons described above.

Notably, axon differentiation occurs at a time when the process is well under a hundred micrometers long. Therefore, there is no readily apparent reason why the expansion of the MT array that occurs during axon differentiation could not be based entirely on local MT assembly fueled by diffusion of tubulin subunits. Nevertheless, our data indicate that this is not the case. The expansion of the MT array at this point in development does not involve an increase in average MT length, but rather the addition of new MTs, presumably arriving by transport from 
the cell body. Consistent with our model, the highest number of the shortest MTs is present within the most proximal region of the axon. In addition, the MT length range starts expanding during axon differentiation, with the appearance of greater numbers of longer MTs but also greater numbers of shorter MTs. Thus, the shift in MT length distribution is already underway, even at the earliest phase of axon growth. These results provide strong support for our model, confirming its predictions even when the axon is sufficiently short that it could theoretically grow by simpler means.

Also relevant to the claboration of the axonal MT array are changes in the organization of MTs within the array. In the differentiating axon, the MTs are spaced more than twice as close to one another as in the minor processes, and become notably less variable in their spacing and more parallel to one another. This is particularly obvious in the hillock and growth cone regions where MTs in the minor processes but not the axon often cross the paths of others as well as the long axis of the process. In the hillock region of the axon, the MTs coalesce in near-parallel fashion from the cell body, and in the growth cone region, they splay apart in a similar fashion. Interestingly, as the axon continues to develop and grow, the MTs retain this high degree of organization (parallel orientation and low variability in the distance of spacing), but take on a notably wider spacing. Available evidence suggests that these differences in MT organization probably relate to differences in microtubuleassociated proteins (MAPs) between minor processes and axons at different stages of development. For example, axons do not differentiate when the expression of tau is curtailed (Caceres and Kosik, 1990), but minor processes appear to be unaffected. On the other hand, disorganized MTs become organized into tight parallel arrays when tau is expressed in non-neuronal cells (Kanai et al., 1989; Baas et al., 1991, 1994; Knops et al., 1991). Nevertheless, however, tau is present in minor processes as well as axons (Dotti et al., 1987; Kosik and Finch, 1987), suggesting that the manner by which MAPs regulate MT organization is complex.

\section{Concluding remarks}

For the past several years, there has been great interest in identifying the cellular and molecular signals that trigger the differentiation of axons and dendrites. Our studies indicate that a key cellular event underlying axon differentiation is a massive influx of MTs into one of the minor processes. We can only speculate on the signal that might trigger this effect. One possibility is that there is an increase in the nucleation and release of MTs from the centrosome, filling the cell body with high numbers of MTs ready for transport. The signal for such an increase may involve MAPs such as tau, which are known to effect MT nucleation and assembly, as well as the regularity and distance of spacing between MTs, which are also altered during axon differentiation. Still another possibility is that critical elements of the MT transport machinery may begin their expression at a key point in neuronal development, cueing axon differentiation. Additional studies will be required to evaluate the merits of these speculations, and further elucidate the mechanisms by which axons differentiate.

\section{References}

Baas PW, Ahmad, FJ (1992) The plus ends of stable microtubules are the exclusive nucleating structures for microtubules in the axon. $J$ Cell Biol 116:1231-1241.

Baas PW, Ahmad FJ (1993) The transport properties of axonal mi- crotubules establish their polarity orientation. J Cell Biol 120:14271437.

Baas PW, Heidemann SR (1986) Microtubule reassembly from nucleating fragments during the regrowth of amputated neurites. J Cell Biol 103:917-927.

Baas PW, Joshi HC (1992) Gamma-tubulin distribution in the neuron: implications for the origins of neuritic microtubules. J Cell Biol 119: 171-178.

Baas PW, Deitch JS, Black MM, Banker GA (1988) Polarity orientation of microtubules in hippocampal neurons: uniformity in the axon and nonuniformity in the dendrite. Proc Natl Acad Sci USA $85: 8335-8339$.

Baas PW, Black MM, Banker GA (1989) Changes in microtubule polarity orientation during the development of hippocampal neurons in culture. J Cell Biol 109:3085-3094.

Baas PW, Picnkowski TP, Kosik KS (1991) Processes induced by tau expression in $\mathrm{Sf9}$ cells have an axon-like microtubule organization. J Cell Biol 115:1333-1344

Baas PW, Pienkowski TP, Cimbalnik KA, Toyama K, Bakalis S, Ahmad FJ, Kosik KS (1994) Tau confers drug-stability but not cold-stability to microtubules in living cells. J Cell Sci 107:135-143.

Banker G, Goslin K (1991) Characterizing and studying neuronal cultures. In: Culturing nerve cells (Banker G, Goslin K, eds), pp 75109. Cambridge, MA: MIT Press.

Banker GA, Waxman AB (1988) Hippocampal neurons generate natural shapes in cell culture. In: Intrinsic determinants of neuronal form and function (Lasek RJ, Black MM, eds), pp 61-82. New York: Liss.

Black MM, Baas PW (1989) The basis of polarity in neurons. Trends Neurosci 12:211-214

Bottenstein J (1985) Growth and differentiation of neural cells in defined media. In: Cell culture in the neurosciences (Bottenstein JE, Sato G, eds), pp 3-44. New York: Plenum.

Bray D, Bunge MB (1981) Serial analysis of microtubules of cultured rat sensory neurons. J Neurocytol 10:589-605.

Burton P (1987) Microtubules of frog olfactory axons: their length and number/axon. Brain Res 409:71-78.

Caceres A, Kosik KS (1990) Inhibition of neurite polarity by tau antisense oligonucleotides in primary cerebellar neurons. Nature 343: $461-463$.

Dotti CG, Banker GA (1987) Experimentally induced alteration in the polarity of developing neurons. Nature 330:254-256.

Dotti CG, Banker GA (1991) Intracellular organization of hippocampal neurons during the development of neuronal polarity. J Cell Sci [Suppl] 15:75-84.

Dotti CG, Banker GA, Binder, LI (1987) The expression and distribution of the microtubule-associated proteins tau and microtubuleassociated protein 2 in hippocampal neurons in the rat brain in situ and in cell culture. Neuroscience 23:121-130.

Dotti CG, Sullivan CA, Banker GA (1988) The establishment of polarity by hippocampal neurons in culture. J Neurosci 8:1454-1468.

Goslin K, Banker G (1989) Experimental observations on the development of polarity by hippocampal neurons in culture. J Cell Biol 108:1507-1516.

Goslin K, Banker G (1991) Rat hippocampal neurons in low-density culture. In: Culturing nerve cells (Banker G, Goslin K, eds), pp 251281. Cambridge, MA: MIT Press.

Joshi HC, Baas PW (1993) A new perspective on microtubules and axon growth. J Cell Biol 121:1191-1196.

Joshi HC, Baas P, Chu DT, Heidemann SR (1986) The cytoskeleton of neurites after microtubule depolymerization. Exp Cell Res 163: 233-245.

Kanai Y, Takemura R, Oshima T, Ihara Y, Yanagisawa M, Masaki T, Hirokawa N (1989) Expression of multiple tau isoforms and microtubule bundle formation in fibroblasts transfected with a single tau cDNA. J Cell Biol 109:1173-1184.

Keith C (1987) Slow transport of tubulin in the neurites of differentiated PC12 cells. Science 235:337-339.

Keith C, Farmer M (1993) Microtubule behavior in PC12 neurites: variable results obtained with photobleach technology. Cell Motil Cytoskel 25:345-357.

Kirschner M, Mitchison T (1986) Beyond self-assembly: from microtubules to morphogenesis. Cell 45:329-342.

Knops J, Kosik KS, Lee G, Pardee JD, Cohen-Gould L, McConlogue L (1991) Overexpression of tau in non-neuronal cells induces long cellular processes. J Cell Biol 114:725-734.

Kosik KS, Finch EA (1987) MAP-2 and tau segregate into axonal and 
dendritic domains after the elaboration of morphologically distinct neurites: an immunocytochemical study of cultured rat cerebrum. J Neurosci 7:3142-3153.

Lasek RJ (1988) Studying the intrinsic determinants of neuronal form and function. In: Intrinsic determinants of neuronal form and function (Lasek RJ, Black MM, eds), pp 1-58. New York: Liss.

Lim S-S, Sammak PJ, Borisy GG (1989) Progressive and spatially differentiated stability of microtubules in developing neuronal cells. J Cell Biol 109:253-264.

Lim S-S, Edson KJ, Letourneau PC, Borisy GG (1990) A test of microtubule translocation during neurite elongation. J Cell Biol 111: 123-130.

Okabe S, Hirokawa N (1989) Turnover of fluorescently labeled tubulin and actin in the axon. Nature 343:479-482.

Okabe S, Hirokawa N (1992) Differential behavior of photoactivated microtubules in growing axons of mouse and frog neurons. J Cell Biol 117:105-120.

Okabe S, Hirokawa N (1993) Do photobleached fluorescent micro- tubules move? Reevaluation of fluorescence laser photobleaching both in vitro and in growing axons of mouse and Xenopus axon. J Cell Biol 120:1177-1186.

Reinsch SS, Mitchison TJ, Kirschner M (1991) Microtubule polymer assembly and transport during axonal elongation. J Cell Biol 115 : 365-379.

Sabry JH, O'Connor TP, Evans L, Toroian-Raymond A, Kirschner M, Bentley D (1991) Microtubule behavior during guidance of pioneer neuron growth cones in situ. J Cell Biol 115:381-395.

Tanaka EM, Kirschner MW (1991) Microtubule behavior in the growth cones of living neurons during axon elongation. J Cell Biol 115:345363.

Tsukita S, Ishikawa H (1981) The cytoskeleton of myelinated axons: serial section study. Biomed Res 2:424-437.

Yu W, Centonze VE, Ahmad FJ, Baas PW (1993) Microtubule nucleation and release from the neuronal centrosome. J Cell Biol 122: 349-359. 